\title{
Associations between Circulating Markers of Cholesterol Homeostasis and Macrovascular Events among Patients Undergoing Hemodialysis
}

\author{
Wen-Chin Lee ${ }^{1} \mathbb{D}$, Wei-Hung Kuo ${ }^{1}$, Sin-Hua Moi ${ }^{2} \mathbb{D}$, Barry Chiu ${ }^{3}$, Jin-Bor Chen ${ }^{1, * \mathbb{D}}$ and Cheng-Hong Yang ${ }^{4,5}(\mathbb{D}$ \\ 1 Division of Nephrology, Department of Internal Medicine, Kaohsiung Chang Gung Memorial Hospital and \\ Chang Gung University College of Medicine, Kaohsiung 83301, Taiwan; leewenchin@gmail.com (W.-C.L.); \\ b8701144@cgmh.org.tw (W.-H.K.) \\ 2 Center of Cancer Program Development, E-Da Cancer Hospital, I-Shou University, Kaohsiung 84001, Taiwan; \\ moi9009@gmail.com \\ 3 Department of Epidemiology, Brown University School of Public Health, Providence, RI 02903, USA; \\ barryprchiu@brown.edu \\ 4 Department of Electronic Engineering, National Kaohsiung University of Science and Technology, \\ Kaohsiung 80778, Taiwan; chyang@cc.kuas.edu.tw \\ 5 Ph.D. Program in Biomedical Engineering, Kaohsiung Medical University, Kaohsiung 80708, Taiwan \\ * Correspondence: chenjb1019@gmail.com
}

check for updates

Citation: Lee, W.-C.; Kuo, W.-H.; Moi, S.-H.; Chiu, B.; Chen, J.-B.; Yang, C.-H. Associations between Circulating Markers of Cholesterol Homeostasis and Macrovascular Events among Patients Undergoing Hemodialysis. Nutrients 2021, 13, 1014. https://doi.org/10.3390/ nu13031014

Academic Editor:

Herminia González-Navarro

Received: 23 February 2021

Accepted: 18 March 2021

Published: 21 March 2021

Publisher's Note: MDPI stays neutral with regard to jurisdictional claims in published maps and institutional affiliations.

Copyright: (c) 2021 by the authors. Licensee MDPI, Basel, Switzerland. This article is an open access article distributed under the terms and conditions of the Creative Commons Attribution (CC BY) license (https:// creativecommons.org/licenses/by/ $4.0 /$ )

\begin{abstract}
Current strategies targeting serum cholesterol bring limited benefits to mortality and macrovascular events prevention among hemodialysis patients. Direct measurements and analysis on circulating markers of cholesterol homeostasis could be promising solutions to this bottleneck. We prospectively enrolled 90 maintenance hemodialysis patients and 9 healthy controls in 2019 for 1 year. We measured circulating desmosterol and lathosterol as markers for cholesterol synthesis and campesterol and sitosterol for cholesterol absorption. At baseline, hemodialysis patients showed higher levels of campesterol $(p=0.023)$ compared to healthy controls. During follow-up, we identified $14(15.4 \%)$ patients who experienced macrovascular events. Comparisons of cholesterol homeostasis markers between cohorts with and without macrovascular events showed no significant differences in markers of cholesterol synthesis or absorption. Using logistic regression analysis, the odds ratio was not statistically significant for the prediction of macrovascular events after full-adjusting for age, sex, diabetes, serum albumin, cholesterol, and triglyceride. We concluded that hemodialysis patients demonstrated higher level of cholesterols absorption, indicated by circulating campesterol compared to healthy subjects. Markers for cholesterol homeostasis were not significantly associated with macrovascular events during a 1-year follow-up. Our results shed light on the novel therapeutic target of modulating cholesterol absorption in HD patients.
\end{abstract}

Keywords: cholesterol homeostasis; hemodialysis; macrovascular events; mortality

\section{Introduction}

Macrovascular events are common in chronic kidney disease (CKD) and hemodialysis (HD) populations [1-3]. These events are the most common causes of death among HD patients [4,5]. Dyslipidemia has long been recognized as a potentially modifiable risk factor in the prevention of macrovascular events in HD patients. However, randomized control trials on cholesterol-lowering drugs fail to show benefits for macrovascular events prevention in HD patients. In addition to current research on cholesterol levels among HD patients, investigations on the alterations of cholesterol homeostasis are crucial to better understand the link between dyslipidemia and macrovascular events in HD patients.

Cholesterol is an insoluble lipid molecule that is involved with the structure and function of cellular membrane bilayers and is a substrate for steroid hormone biosynthesis. A complex network is required to maintain intracellular cholesterol homeostasis in cholesterol 
biosynthesis, uptake, efflux, conversion, esterification, and trafficking [6,7]. Various cells have the capacity to regulate cellular free cholesterol concentrations through endogenous synthesis, intracellular esterification, and excretion [8]. Whole-body cholesterol content is regulated by balancing input (intestinal absorption of dietary and biliary cholesterol) and output (hepatic and extra-hepatic synthesis) $[9,10]$. Various circulating markers have been assigned to reflect endogenous cholesterol synthesis (lathosterol, desmosterol, mevalonate, and squalene), intestinal cholesterol absorption (sitosterol, campesterol, and cholestanol), or bile acid synthesis (7alpha-hydroxy-4-cholesten-3-one) in population study [11-15].

There has been limited research on cholesterol homeostasis in CKD and HD population. In an earlier study among dialysis patients with a small sample size, both plasma biomarkers of cholesterol intestinal absorption (campesterol) and endogenous cholesterol synthesis (lathosterol) were reduced compared to healthy participants [16]. Another study showed higher levels of cholesterol intestinal absorption markers (cholestanol) and lower levels of endogenous cholesterol synthesis markers (lathosterol) in HD patients when compared to healthy controls [17].

Considering the lack of a comprehensive assessment of cholesterol homeostasis in HD patients, we performed the present study to examine cholesterol homeostasis via direct measurement of circulating markers of cholesterol intestinal absorption and endogenous cholesterol synthesis. This quantification of circulating biomarkers for cholesterol homeostasis is warranted. The traditional lipid profile is usually derived rather than directly measured. In contrast, mass spectrometry-based cholesterol profiling can accurately measure circulating markers of cholesterol homeostasis. This approach allows researchers to detect alterations in various metabolic pathways that control cholesterol homeostasis. Furthermore, we assessed the association between circulating markers of cholesterol homeostasis and macrovascular events in HD patients.

\section{Materials and Methods}

\subsection{Study Design and Participants}

Adult patients (>18 years) who underwent maintenance HD (4-h thrice weekly) for at least 3 months in the outpatient clinic in Kaohsiung Chang Gung Memorial Hospital in Taiwan from August 2019 to September 2019 were enrolled in this study. All participants underwent HD with appropriate dialyzers (Supplementary Table S1) [18] and bicarbonate-based dialysate, dialysate sodium $140 \mathrm{mEq} / \mathrm{L}$, calcium $3.0 \mathrm{mEq} / \mathrm{L}$, and potassium $2.0 \mathrm{mEq} / \mathrm{L}$. Blood flow rate was $250-300 \mathrm{cc} / \mathrm{min}$ and dialysate flow rate was $500 \mathrm{cc} / \mathrm{min}$. None of the participants received hemodiafiltration. The exclusion criteria were as follows: ongoing treatment for malignancy, acute inflammatory diseases, hospitalization within 3 months, malnutrition defined by serum albumin level $<3.5 \mathrm{~g} / \mathrm{dL}$, pregnancy, and using lipid-lowering drug treatment at least 3 months prior to the study. All participants did not have pre-existing macrovascular events and were followed to September 2020. Healthy controls were recruited voluntarily in the outpatient clinic by posted protocol notification. Informative patient data, including demographic profiles, laboratory parameters, and macrovascular events in the study period were collected.

\subsection{Definition and Assessment of Macrovascular Events}

Macrovascular events were defined as occurrence of angina pectoris, unstable angina, myocardial infarction, cardiac catheterization-proved coronary artery insufficiency, cerebrovascular accident, or transient ischemic attack. Information of macrovascular events was confirmed through medical records from the study clinic and hospitalization records.

\subsection{Biochemistry}

All blood samples from HD participants in the fasting status and in mid-week (Wednesday and Thursday) were obtained. Blood samples for biochemistry measurement were obtained using commercial kits and an autoanalyzer (Hitachi 7600-210, Hitachi Ltd., Tokyo, Japan). Albumin levels were measured using the bromocresol green method. The plasma 
total cholesterol, triglyceride, and high-density lipoprotein cholesterol (HDL-C) levels were determined enzymatically on the Eroset Hitachi 7600-210 analyzer. The low-density lipoprotein cholesterol (LDL-C) levels were calculated using to the Friedewald formula [19], which provides reliable values up to a triglyceride level of $4.0 \mathrm{mmol} / \mathrm{L}$.

\subsection{Measurements of Circulating Markers of Cholesterol Homeostasis}

Concentrations of desmosterol, lathosterol, campesterol, and sitosterol were quantified using a gas chromatography-mass spectrometry method similar to prior studies [20]. Briefly, plasma samples from HD patients and healthy controls were added with $10 \mathrm{uL}$ 5-alphaCholestane in the standard solution, and potassium hydroxide standard for saponification for $1 \mathrm{~h}$ after centrifugation. After saponification, the solution was added with n-Hexane. Later, the derivatization reagents (BSTFA-TMCS = 99:1) were added to the residue. After the derivatization reaction, the samples were added with the standard solution according to the preparation of standard solution tables provided by the commercial company (Ardent BioMed LLC, Mt. View, CA, USA). Linear regression was used to quantitatively determine the individual standard curve according to internal quantitative ion area ratio (x) and compound concentration (y). Since the non-cholesterol sterols are transported in plasma by lipoproteins, their concentrations were expressed relative to the concentration of total cholesterol ( $\mathrm{mmol} / \mathrm{mol}$ of cholesterol) to correct for the differing number of lipoprotein acceptor particles. Cholesterol metabolic balance score was calculated as desmosterol + lathosterol/campesterol + sitosterol. We defined the cholesterol metabolic balance scores as follows: score >1.1, increased endogenous synthesis; 0.5-1.1, normal metabolism; and $0.2-0.5$, increased intestinal absorption.

\subsection{Statistical Analysis}

Baseline demographic characteristics and laboratory measurements among HD patients and healthy controls are presented as frequency (percentage) and mean (standard deviation). The distribution difference was estimated using the independent two-sample t-test, Mann-Whitney, chi-square, or Fisher's exact test. Logistic regression analysis was performed to evaluate the association between macrovascular events and baseline characteristics in HD patients. The correlation between the cholesterol absorption and synthesis parameters and associated variables was estimated using the Pearson correlation test. The cholesterol absorption and synthesis parameters, which included desmosterol, lathosterol, campesterol, sitosterol, and cholesterol metabolic balance scores, were included in the univariate logistic regression model. All included variables were retained in the fully adjusted logistic regression model. Demographic characteristics including age, sex, diabetes, albumin, cholesterol, and triglyceride were involved as covariates. All $p$-values were two-sided, and $p<0.05$ was considered statistically significant. All statistical analyses were performed using the R 4.0.3 software (R Core Team, 2020 R Foundation for Statistical Computing, Vienna, Austria).

\section{Results}

We pre-screened potentially eligible participants $(n=115)$ who had received maintenance HD in outpatient clinic in August 2019. Ten patients expressed not to participate this study owing to individual reason. Thus, 105 HD patients entered our screening stage. After implementing our selection criteria, 91 patients were enrolled in the study. In a 1-year follow-up, 90 patients completed the study (Figure 1). Nine healthy volunteers were also recruited at the baseline period. 


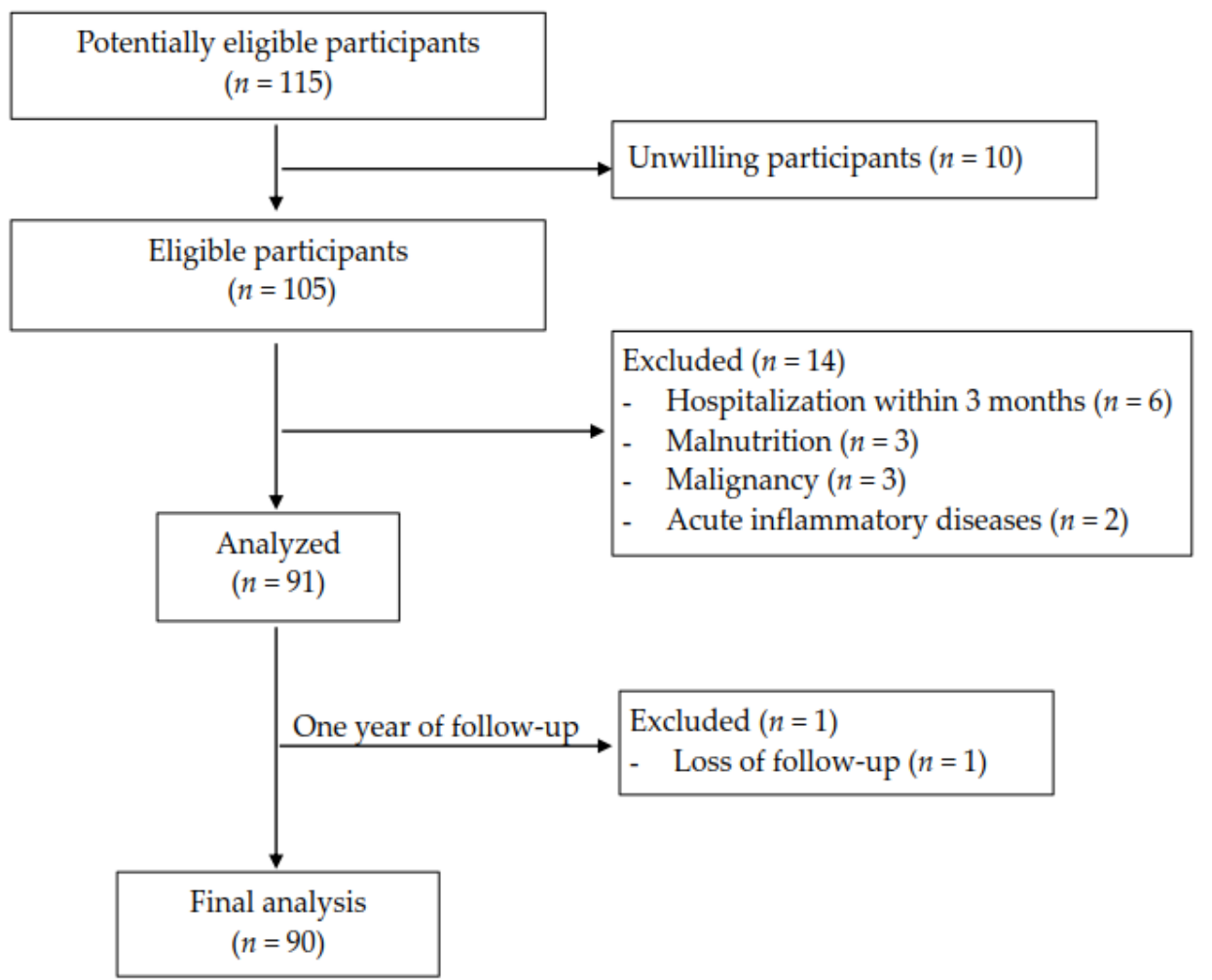

Figure 1. Study design and flowchart of patient selection.

\subsection{Characteristics of Circulating Markers of Cholesterol Homeostasis in HD Patients}

Baseline characteristics in the study cohort are shown in the Table 1 . The mean age was 65 years, and gender distribution was similar within the HD cohort. HD patients showed lower levels of desmosterol $(p=0.523)$ and lathosterol $(p=0.499)$ and higher levels of campesterol $(p=0.023)$ and sitosterol $(p=0.199)$ compared to healthy controls. Cholesterol metabolic balance scores were lower in HD cohort compared to healthy controls, though the difference was not statistically significant $(p=0.109)$.

Table 1. Baseline characteristics in hemodialysis (HD) patients and healthy controls $(n=99)$.

\begin{tabular}{cccc}
\hline Variables & $\begin{array}{c}\text { HD } \\
(\boldsymbol{n}=\mathbf{9 0})\end{array}$ & $\begin{array}{c}\text { Healthy } \\
\text { Control }(\boldsymbol{n}=\mathbf{9})\end{array}$ & $\boldsymbol{p}$ \\
\hline Age, years & $65 \pm 7$ & $62 \pm 11$ & 0.196 \\
Sex & & & 1.000 \\
Female & $45(50.0 \%)$ & $4(44.4 \%)$ & \\
Male & $45(50.0 \%)$ & $5(55.6 \%)$ & \\
BMI, kg/m ${ }^{2}$ & $22.6 \pm 4.0$ & $22.1 \pm 3.1$ & 0.766 \\
Systolic blood pressure, mmHg & $137 \pm 30$ & $\mathrm{~N} / \mathrm{A}$ & \\
Diastolic blood pressure, mmHg & $67 \pm 14$ & $\mathrm{~N} / \mathrm{A}$ & - \\
Dialysis vintage, years & $10.4 \pm 7.49$ & $0(0 \%)$ & - \\
Diabetes & $16(17.8 \%)$ & & - \\
Etiologies of renal failure & & $\mathrm{N} / \mathrm{A}$ & $\mathrm{N} / \mathrm{A}$ \\
Primary kidney disease & $37(41.1 \%)$ & $\mathrm{N} / \mathrm{A}$ & \\
Systemic disease & $37(41.1 \%)$ & $65.2 \pm 39.9$ & 0.523 \\
Others & $16(17.8 \%)$ & $157.2 \pm 136.3$ & 0.499 \\
Desmosterol, $10^{2}$ mmol/mol cholesterol & $61.6 \pm 86.5$ & $207.9 \pm 91.4$ & 0.023 \\
Lathosterol, $10^{2}$ mmol/mol cholesterol & $111.6 \pm 95.9$ & $168.3 \pm 89.0$ & 0.199 \\
Campesterol, $10^{2}$ mmol/mol cholesterol & $330.0 \pm 179.5$ & $0.7 \pm 0.6$ & 0.109 \\
Sitosterol, $10^{2}$ mmol/mol cholesterol & $218.4 \pm 128.4$ & & \\
Cholesterol metabolic balance score & $0.4 \pm 0.3$ & & \\
\hline
\end{tabular}


Table 1. Cont.

\begin{tabular}{cccc}
\hline Variables & $\begin{array}{c}\text { HD } \\
(\boldsymbol{n}=\mathbf{9 0})\end{array}$ & $\begin{array}{c}\text { Healthy } \\
\text { Control }(\boldsymbol{n}=\mathbf{9 )}\end{array}$ & $\boldsymbol{p}$ \\
\hline Laboratory measurements & & & \\
Hemoglobin, g/dL & $10.7 \pm 1.3$ & $13.5 \pm 1.9$ & $<0.001$ \\
Albumin, g/dL & $3.9 \pm 0.3$ & $4.3 \pm 0.3$ & 0.015 \\
BUN, mg/dL * & $69.0(57.0-83.0)$ & $14.0(13.0-16.0)$ & $<0.001$ \\
Cr, $\mathrm{mg} / \mathrm{dL}$ & $10.4 \pm 2.1$ & $1.0 \pm 0.4$ & $<0.001$ \\
Total Cholesterol, $\mathrm{mg} / \mathrm{dL}^{*}$ & $153.5(136.0-183.0)$ & $191.0(177.0-199.0)$ & 0.005 \\
Triglyceride, $\mathrm{mg} / \mathrm{dL}$ * & $114.5(84.0-165.0)$ & $117.0(84.0-131.0)$ & 0.789 \\
HDL-C, $\mathrm{mg} / \mathrm{dL}$ & $45.4 \pm 15.3$ & $52.1 \pm 9.6$ & 0.049 \\
LDL-C, $\mathrm{mg} / \mathrm{dL}$ & $90.2 \pm 34.6$ & $122.3 \pm 41.1$ & 0.005 \\
\hline
\end{tabular}

Abbreviations: HD—hemodialysis; BMI—body mass index; BUN—blood urea nitrogen; $\mathrm{Cr}-$ creatinine HDL-high-density lipoprotein; LDL-low-density lipoprotein; C—cholesterol; ${ }^{*}$ median (interquartile range).

\subsection{Differences between HD Patients with and without Macrovascular Events}

Patients who had macrovascular events in the study period were significantly older in age $(p<0.001)$, had higher body mass index $(p<0.001)$, and longer dialysis vintage $(p<0.001)$ compared to those without events. Although patients with macrovascular events showed lower levels of intestinal cholesterol absorption, the differences did not reach statistical significance. Cholesterol metabolic balance scores were similar between two groups (Table 2).

Table 2. Baseline characteristics in HD patients with and without macrovascular events $(n=90)$.

\begin{tabular}{cccc}
\hline Variables & $\begin{array}{c}\text { With Events } \\
(\boldsymbol{n}=\mathbf{1 4})\end{array}$ & $\begin{array}{c}\text { Without Events } \\
(\boldsymbol{n}=\mathbf{7 6})\end{array}$ & $p$ \\
\hline Age, years & $69 \pm 8$ & $64 \pm 6$ & $<0.001$ \\
Sex & & & 0.144 \\
Female & $4(28.6 \%)$ & $41(53.9 \%)$ & \\
Male & $10(71.4 \%)$ & $35(46.1 \%)$ & \\
BMI, kg/m ${ }^{2}$ & $24.0 \pm 6.0$ & $22.3 \pm 3.3$ & $<0.001$ \\
Systolic blood pressure, mmHg & $136 \pm 30$ & $154 \pm 24$ & 0.094 \\
Diastolic blood pressure, mmHg & $67 \pm 15$ & $68 \pm 10$ & 0.528 \\
Dialysis vintage, years & $11.4 \pm 9.1$ & $10.2 \pm 7.2$ & $<0.001$ \\
Diabetes & $3(21.4 \%)$ & $13(17.1 \%)$ & 0.709 \\
Desmosterol, 10 ${ }^{2}$ mmol/mol cholesterol & $61.7 \pm 49.6$ & $61.5 \pm 91.9$ & 0.925 \\
Lathosterol, 10² mmol/mol cholesterol & $99.1 \pm 108.0$ & $113.9 \pm 94.1$ & 0.214 \\
Campesterol, 10 ${ }^{2}$ mmol/mol cholesterol & $298.1 \pm 139.7$ & $335.9 \pm 186.0$ & 0.652 \\
Sitosterol, 10 ${ }^{2}$ mmol/mol cholesterol & $199.2 \pm 83.5$ & $221.9 \pm 135.2$ & 0.854 \\
Cholesterol metabolic balance score & $0.4 \pm 0.4$ & $0.4 \pm 0.3$ & 0.544 \\
Laboratory measurements & & & \\
Hemoglobin, g/dL & $10.7 \pm 1.3$ & $10.7 \pm 1.3$ & 0.982 \\
Albumin, g/dL & $3.9 \pm 0.4$ & $3.9 \pm 0.3$ & 0.551 \\
BUN, mg/dL & $75.0(64.0-84.0)$ & $68.0(56.0-80.0)$ & 0.319 \\
Cr, mg/dL & $10.5 \pm 2.3$ & $10.4 \pm 2.0$ & 0.555 \\
Total Cholesterol, mg/dL * & $141.5(137.0-164.0)$ & $158.0(136.0-186)$ & 0.157 \\
Triglyceride, mg/dL * & $110.0(100.0-136.0)$ & $117.0(82.0-167.0)$ & 0.925 \\
HDL-C, mg/dL & $39.9 \pm 11.7$ & $46.4 \pm 15.7$ & 0.148 \\
LDL-C, mg/dL & $81.1 \pm 20.0$ & $91.9 \pm 36.6$ & 0.395 \\
\hline
\end{tabular}

Abbreviations: BMI—body mass index; BUN—blood urea nitrogen; $\mathrm{Cr} —$ creatinine; HDL—high-density lipoprotein; LDL—low-density lipoprotein; $\mathrm{C}$-cholesterol; * median (interquartile range).

\subsection{Correlations of Cholesterol Homeostasis Markers}

In correlation analysis, body mass index showed a significantly positive correlation between lathosterol and cholesterol metabolic balance score and negative between campesterol and sitosterol. The association between cholesterol metabolic balance score and cholesterol synthesis markers was significantly positive. Conversely, the association be- 
tween cholesterol intestinal absorption markers was significantly negative. In addition, total cholesterol showed a significantly negative correlation with lathosterol. Triglyceride showed a significantly negative correlation with campesterol and sitosterol. Regarding lipoprotein profiles, HDL-C showed significantly positive correlation with sitosterol. LDLC showed significantly negative correlation with lathosterol (Table 3).

Table 3. Pearson correlation analysis between studied continuous variables.

\begin{tabular}{|c|c|c|c|c|c|}
\hline Variables & Desmosterol & Lathosterol & Campesterol & Sitosterol & $\begin{array}{c}\text { Cholesterol } \\
\text { Metabolic } \\
\text { Balance Score }\end{array}$ \\
\hline Age & -0.15 & -0.18 & -0.16 & -0.13 & -0.08 \\
\hline BMI & -0.07 & $0.32 * *$ & $-0.21 *$ & $-0.30^{* *}$ & $0.33^{* *}$ \\
\hline Desmosterol & - & $0.40^{* * * *}$ & 0.18 & 0.12 & $0.42 * * * *$ \\
\hline Lathosterol & $0.40^{* * * *}$ & - & 0.07 & -0.02 & $0.65^{* * * *}$ \\
\hline Campesterol & 0.18 & 0.07 & - & $0.85^{* * * *}$ & $-0.40^{* * *}$ \\
\hline Sitosterol & 0.12 & -0.02 & $0.85^{* * * *}$ & - & $-0.42^{* * * *}$ \\
\hline $\begin{array}{c}\text { Cholesterol metabolic } \\
\text { balance score }\end{array}$ & $0.42^{* * * *}$ & $0.65^{* * * *}$ & $-0.40^{* * *}$ & $-0.42^{* * * *}$ & - \\
\hline Albumin & -0.07 & 0.14 & -0.11 & -0.03 & 0.08 \\
\hline Total Cholesterol & -0.17 & $-0.23 *$ & -0.18 & -0.16 & -0.11 \\
\hline Triglyceride & -0.18 & 0.06 & $-0.28 * *$ & $-0.32^{* *}$ & 0.13 \\
\hline HDL-C & 0.15 & -0.14 & 0.18 & $0.22 *$ & -0.18 \\
\hline LDL-C & -0.19 & $-0.23 *$ & -0.16 & -0.14 & -0.11 \\
\hline
\end{tabular}

\subsection{Predictions of Macrovascular Events in HD Patients by Cholesterol Homeostasis Markers}

Results from unadjusted and adjusted models for macrovascular events prediction using logistic regression analysis are shown in Table 4. Overall, individual markers for cholesterol synthesis and absorption and cholesterol metabolic balance score did not show significant odds ratio (OR) for macrovascular events prediction.

Table 4. Logistic regression for macrovascular events prediction using cholesterol homeostasis markers.

\begin{tabular}{ccccc}
\hline \multirow{2}{*}{ Variables } & \multicolumn{2}{c}{ Unadjusted } & \multicolumn{2}{c}{ Full-Adjusted } \\
\cline { 2 - 5 } & OR (95\% CI) & $p$ & OR (95\% CI) & $p$ \\
\hline Desmosterol & $1.000(0.989-1.006)$ & 0.994 & $0.997(0.968-1.008)$ & 0.699 \\
Lathosterol & $0.998(0.989-1.004)$ & 0.598 & $0.997(0.979-1.011)$ & 0.697 \\
Campesterol & $0.999(0.995-1.002)$ & 0.470 & $1.000(0.988-1.011)$ & 0.939 \\
Sitosterol & $0.998(0.992-1.003)$ & 0.544 & $1.001(0.985-1.015)$ & 0.898 \\
Cholesterol metabolic balance score & $1.012(0.132-4.993)$ & 0.989 & $9.003(0.013-4621.542)$ & 0.467 \\
\hline
\end{tabular}

Full-adjusted model includes age, sex, diabetes, albumin, cholesterol, and triglyceride as covariates.

\section{Discussion}

Cholesterol homeostasis is complex in HD patients. Strategies simply to decrease LDL-C, as seen in the 4D (Die Deutsche Diabetes Dialyse Studie) and AURORA (A Study to Evaluate the Use of Rosuvastatin in Subjects on Regular Hemodialysis), failed to show a survival benefit in this population despite leading to an LDL cholesterol reduction in a magnitude shown to significantly reduce cardiovascular morbidity and mortality in the general population [21-23]. Cholesterol homeostasis involves hepatic and extra-hepatic endogenous synthesis and intestinal and biliary absorption of exogenous cholesterol. Animal-derived cholesterol and plant-derived phytosterols or plant sterols are the main component of dietary sterols. Among plant sterols, sitosterol and campesterol are most abundant. An observation from inherited disease patients with ABCG5 and/or ABCG8 deficiency presented sitosterolemia and premature coronary disease [24,25]. This phenotypical observation leads to a hypothesis that high levels of plant sterols are atherogenic. 
However, data from prior studies did not reveal consistent association between coronary heart disease and plant sterols. The risk of incident myocardial infarction in men increased 1.8-fold for sitosterol in the Prospective Cardiovascular Munster study [26]. The Framingham Offspring Study reported that campesterol, sitosterol, and cholestanol were significantly associated with cardiovascular disease [27]. Additionally, several small-scale studies reported that higher levels of cholesterol absorption markers increased the risk for cardiovascular diseases [28,29]. In contrast, the EPIC-Norfolk study and the Longitudinal Aging Study Amsterdam (LASA) study both reported that increased cholesterol absorption markers were associated with decreased risk for cardiovascular disease [30,31]. Moreover, a study reported no significant association between sitosterol and campesterol and coronary atherosclerosis among middle-aged men and women [32]. Recently, a study reported that a low serum lathosterol is correlated with an increased risk for cardiovascular events and an excess of all-cause mortality across 4.9 years of follow-up. None of the other cholesterol homeostasis markers demonstrated this relationship [33]. Various factors may account for the variability in the above studies, such as study design and selection bias, diverse participants in age, health, and genetic background, individual lipoprotein profile, gut microbiome, and variable experimental methods to measure concentration of plant sterols. Given the above observation, a standardization of experimental methods and large-scale longitudinal study is needed to clarify this controversial issue.

There are only a few studies that address cholesterol homeostasis and its relationship with cardiovascular risk within CKD or HD populations. CKD patients were reported to have impaired intestinal fat absorption [34]. In a study on eight HD patients, investigators reported that HD patients demonstrated significantly lower fractional cholesterol synthesis (lathosterol) and lower lathosterol to cholesterol ratio compared to healthy controls [16]. Investigators concluded that hepatic cholesterol synthesis was impaired in HD patients since the ratio of lathosterol to cholesterol reflecting hepatic activity of HMG-CoA reductase and fecal total hepatic cholesterol balance synthesis $[16,35,36]$. Later, a study reported that HD patients had lower lathosterol and higher cholestanol levels compared to healthy controls [17]. Results of our study were in line with previous studies. We found that HD patients demonstrated lower levels of cholesterol synthesis markers (lathosterol and desmosterol) and higher levels of cholesterol absorption markers (campesterol and sitosterol) compared to healthy controls. Our analysis using cholesterol metabolic balance scores also indicated increased cholesterol absorption in HD patients. These results reminded us of the cardiovascular protection effects shown within the SHARP (the Study of Heart and Renal Protection) study in which significant reduction in cardiovascular events in CKD and HD is achieved by combining inhibition of cholesterol synthesis via statin treatment and inhibition of intestinal cholesterol absorption via ezetimibe [37].

In the present study, we found that body mass index demonstrated a significantly positive correlation with lathosterol and a negative association with campesterol and sitosterol. Although the exact mechanisms were not clear, our results were partly supported from research on insulin resistance, metabolic syndrome, and diabetes [38-42]. Patients with these insulin resistance-mediated metabolic derangements exhibited a high cholesterol synthesis and low cholesterol absorption profile. Greater cholesterol synthesis in the liver would lead to less cholesterol absorption. Another interesting finding in our study was that lathosterol demonstrated significantly negative correlation with total cholesterol and LDLC. On the contrary, campesterol and sitosterol demonstrated a positive correlation with HDL-C and a negative correlation with triglyceride. Considering a reciprocal relationship between cholesterol synthesis and absorption, it is reasonable to explain our correlation results. Notably, several food products have been added with plant sterols for their effects on reducing blood cholesterol level. This in turn reduces cholesterol absorption in the intestine. Consequently, blood levels of LDL-C are reduced because of foods enriched with plant sterols [43]. We did not collect dietary information of our participants. Therefore, the influence of dietary plant sterols on correlation analysis cannot be obtained in our statement. 
Interestingly, the association between cholesterol homeostasis and mortality is rarely reported for CKD and HD populations. Rogacev et al. reported that cholestanol above median levels had a 2.24-fold risk for all-cause mortality across 3.4 years in HD patients. However, lower lathosterol levels did not demonstrate a similar relationship [17]. In our study, we also did not find the associations between cholesterol synthesis and absorption markers with macrovascular events after adjusting for potential confounding factors in our HD cohort. However, our results were limited by the small number of composite events and a short follow-up period. Furthermore, our study cohort presented reasonable control in their blood pressure, HDL-C, and LDL-C levels. A plausible explanation may be rooted in these favorable clinical situations. Non-traditional risk factors are known to play crucial roles in macrovascular events in HD patients $[44,45]$, but the weights of impacts between these factors and cholesterol homeostasis markers remain unknown. An additional explanation for the lack of association between cholesterol homeostasis markers and macrovascular events in our study might be that non-traditional risk factors outweigh the cholesterol homeostasis markers in macrovascular events in HD patients. To overcome our limitations and to clarify these issues, a further large-scale study with thorough analysis of traditional and non-traditional risk factors and longer follow-up periods is required.

Our study has some limitations. First, the observational design does not completely exclude the possibility of potential confounders that influence the observed outcomes. Although some confounders such as age, blood pressure, and diabetes were adjusted in our study, we agree that some confounders may partly contribute to the composite outcomes. Second, our dataset lacked relevant information known to influence cholesterol homeostasis such as dietary records, insulin sensitivity assessment, specific genotypes, and genetic polymorphisms. Therefore, we cannot exclude the influence of these unmeasured factors. Third, we did not consider the variation in diurnal and time changes in cholesterol homeostasis. Although we used cholesterol metabolic balance scores to diminish the influence of these drawbacks, the validation of our methods warrants further testing through a large-scale population study. Fourth, our study cohort was predominantly of Asian ethnicity and may have a unique lipoprotein profile. Care must be taken when our results are extrapolated to other ethnicities and disease states. Finally, the number of participants in our study was relatively small and composite events were also small. Furthermore, the follow-up duration was only 1 year. These considerations may diminish statistical power in our results. However, our study remains strength in understanding the impact of cholesterol homeostasis on macrovascular events in HD population. To our knowledge, our study is the first report of the relationship between cholesterol homeostasis and macrovascular events in an Asian HD population. In addition, our study could be a proxy to further investigate the diverse cholesterol homeostasis profiles among dialysis populations. Therefore, effective therapeutic measures could be possible to prevent vascular events in HD populations by considering cholesterol absorption and synthesis profiles.

\section{Conclusions}

In conclusion, our study demonstrated increased circulating campesterol and a trend of increased cholesterol intestinal absorption and reduced cholesterol synthesis in HD patients. Although this cholesterol homeostasis pattern was not directly associated with macrovascular events across a 1-year observational period, it sheds light on the novel therapeutic target of modulating cholesterol absorption in HD patients.

Supplementary Materials: The following are available online at https:/ / www.mdpi.com/2072-664 3/13/3/1014/s1, Table S1: Distribution of dialyzers and diabetes in HD cohort $(n=90)$.

Author Contributions: Conceptualization, J.-B.C.; methodology, J.-B.C., W.-H.K. and W.-C.L.; software, S.-H.M.; validation, J.-B.C., B.C. and S.-H.M.; formal analysis, S.-H.M.; investigation, J.-B.C. and W.-H.K.; resources, J.-B.C. data curation, J.-B.C., B.C. and S.-H.M.; writing-original draft preparation, J.-B.C. and W.-C.L.; writing-review and editing, J.-B.C., B.C. and W.-C.L.; visualization, J.-B.C.; 
supervision, C.-H.Y.; project administration, J.-B.C.; funding acquisition, J.-B.C. All authors have read and agreed to the published version of the manuscript.

Funding: This research was funded by Kaohsiung Chang Gung Memorial Hospital, Taiwan, grant number CMRPG8K0541.

Institutional Review Board Statement: The study was conducted according to the guidelines of the Declaration of Helsinki and approved by the Institutional Review Board of Kaohsiung Chang Gung Memorial Hospital, Taiwan (protocol code 201901496B0 and date of approval was 1 October 2019).

Informed Consent Statement: Informed consent was obtained from all subjects involved in the study.

Data Availability Statement: The data presented in this study are available on request from the corresponding author.

Acknowledgments: All authors were grateful to the expert team at the Super Micro Mass Research and Technology Center, Cheng Shiu University, Kaohsiung, Taiwan for their experimental assistance.

Conflicts of Interest: The authors declare no conflict of interest. The funders had no role in the design of the study; in the collection, analyses, or interpretation of data; in the writing of the manuscript, or in the decision to publish the results.

\section{References}

1. Hou, F.; Jiang, J.; Chen, J.; Yu, X.; Zhou, Q.; Chen, P.; Mei, C.; Xiong, F.; Shi, W.; Zhou, W.; et al. China collaborative study on dialysis: A multi-centers cohort study on cardiovascular diseases in patients on maintenance dialysis. BMC Nephrol. 2012, 13, 94. [CrossRef]

2. Wang, H.H.; Hung, S.Y.; Sung, J.M.; Hung, K.Y.; Wang, J.D. Risk of stroke in long-term dialysis patients compared with the general population. Am. J. Kidney Dis 2014, 63, 604-611. [CrossRef] [PubMed]

3. Lee, W.-C.; Lee, Y.-T.; Li, L.-C.; Ng, H.-Y.; Kuo, W.-H.; Lin, P.-T.; Liao, Y.-C.; Chiou, T.T.-Y.; Lee, C.-T. The Number of Comorbidities Predicts Renal Outcomes in Patients with Stage 3-5 Chronic Kidney Disease. J. Clin. Med. 2018, 7, 493. [CrossRef]

4. De Jager, D.J.; Grootendorst, D.C.; Jager, K.J.; Van Dijk, P.C.; Tomas, L.M.J.; Ansell, D.; Collart, F.; Finne, P.; Heaf, J.G.; De Meester, J.; et al. Cardiovascular and Noncardiovascular Mortality Among Patients Starting Dialysis. JAMA 2009, 302, 1782-1789. [CrossRef] [PubMed]

5. United States Renal Data System. 2020 USRDS Annual Data Report: Epidemiology of Kidney Disease in the United States; National Institutes of Health, National Institute of Diabetes and Digestive and Kidney Diseases: Bethesda, MD, USA, 2020.

6. Chang, T.Y.; Chang, C.C.; Ohgami, N.; Yamauchi, Y. Cholesterol sensing, trafficking, and esterification. Annu. Rev. Cell Dev. Biol. 2006, 22, 129-157. [CrossRef]

7. Tabas, I. Consequences of cellular cholesterol accumulation: Basic concepts and physiological implications. J. Clin. Investig. 2002, 110, 905-911. [CrossRef]

8. Ikonen, E. Cellular cholesterol trafficking and compartmentalization. Nat. Rev. Mol. Cell Biol. 2008, 9, 125-138. [CrossRef] [PubMed]

9. Kruit, J.K.; Groen, A.K.; van Berkel, T.J.; Kuipers, F. Emerging roles of the intestine in control of cholesterol metabolism. World J. Gastroenterol. 2006, 12, 6429-6439. [CrossRef]

10. Cohen, D.E. Balancing cholesterol synthesis and absorption in the gastrointestinal tract. J. Clin. Lipidol. 2008, 2, S1-S3. [CrossRef]

11. Miettinen, T.A.; Tilvis, R.S.; Kesäniemi, Y.A. Serum cholestanol and plant sterol levels in relation to cholesterol metabolism in middle-aged men. Metabolism 1989, 38, 136-140. [CrossRef]

12. Miettinen, T.A.; Tilvis, R.S.; Kesäniemi, Y.A. Serum plant sterols and cholesterol precursors reflect cholesterol absorption and synthesis in volunteers of a randomly selected male population. Am. J. Epidemiol. 1990, 131, 20-31. [CrossRef]

13. Simonen, P.; Gylling, H.; Miettinen, T.A. The validity of serum squalene and non-cholesterol sterols as surrogate markers of cholesterol synthesis and absorption in type 2 diabetes. Atherosclerosis 2008, 197, 883-888. [CrossRef]

14. Matthan, N.R.; Raeini-Sarjaz, M.; Lichtenstein, A.H.; Ausman, L.M.; Jones, P.J. Deuterium uptake and plasma cholesterol precursor levels correspond as methods for measurement of endogenous cholesterol synthesis in hypercholesterolemic women. Lipids 2000, 35, 1037-1044. [CrossRef]

15. Sauter, G.; Berr, F.; Beuers, U.; Fischer, S.; Paumgartner, G. Serum concentrations of 7alpha-hydroxy-4-cholesten-3-one reflect bile acid synthesis in humans. Hepatology 1996, 24, 123-126. [CrossRef]

16. Igel-Korcagova, A.; Raab, P.; Brensing, K.A.; Pöge, U.; Klehr, H.U.; Igel, M.; von Bergmann, K.; Sudhop, T. Cholesterol metabolism in patients with chronic renal failure on hemodialysis. J. Nephrol. 2003, 16, 850-854.

17. Rogacev, K.S.; Pinsdorf, T.; Weingärtner, O.; Gerhart, M.K.; Welzel, E.; van Bentum, K.; Popp, J.; Menzner, A.; Fliser, D.; Lütjohann, D.; et al. ; et al. Cholesterol synthesis, cholesterol absorption, and mortality in hemodialysis patients. Clin. J. Am. Soc. Nephrol. 2012, 7, 943-948. [CrossRef]

18. Haroon, S.; Davenport, A. Choosing a dialyzer: What clinicians need to know. Hemodial. Int. 2018, 22, S65-S74. [CrossRef] [PubMed] 
19. Knopfholz, J.; Disserol, C.C.D.; Pierin, A.J.; Schirr, F.L.; Streisky, L.; Takito, L.L.; Ledesma, P.M.; Faria-Neto, J.R.; Olandoski, M.; Da Cunha, C.L.P.; et al. Validation of the Friedewald Formula in Patients with Metabolic Syndrome. Cholesterol 2014, $2014,261878$. [CrossRef] [PubMed]

20. Matthan, N.R.; Giovanni, A.; Schaefer, E.J.; Brown, B.G.; Lichtenstein, A.H. Impact of simvastatin, niacin, and/or antioxidants on cholesterol metabolism in cad patients with low hdl. J. Lipid Res. 2003, 44, 800-806. [CrossRef]

21. Fellstrom, B.C.; Jardine, A.G.; Schmieder, R.E.; Holdaas, H.; Bannister, K.; Beutler, J.; Chae, D.W.; Chevaile, A.; Cobbe, S.M.; Gronhagen-Riska, C.; et al. Rosuvastatin and cardiovascular events in patients undergoing hemodialysis. N. Engl. J. Med. 2009, 360, 1395-1407. [CrossRef] [PubMed]

22. Wanner, C.; Krane, V.; Marz, W.; Olschewski, M.; Mann, J.F.; Ruf, G.; Ritz, E.; German, D.; Dialysis Study, I. Atorvastatin in patients with type 2 diabetes mellitus undergoing hemodialysis. N. Engl. J. Med. 2005, 353, 238-248. [CrossRef]

23. Cholesterol Treatment Trialists' (CTT) Collaboration. Efficacy and safety of more intensive lowering of LDL cholesterol: A meta-analysis of data from 170,000 participants in 26 randomised trials. Lancet 2010, 376, 1670-1681. [CrossRef]

24. Berge, K.E.; Tian, H.; Graf, G.A.; Yu, L.; Grishin, N.V.; Schultz, J.; Kwiterovich, P.; Shan, B.; Barnes, R.; Hobbs, H.H. Accumulation of dietary cholesterol in sitosterolemia caused by mutations in adjacent abc transporters. Science 2000, 290, 1771-1775. [CrossRef]

25. Lee, M.H.; Lu, K.; Hazard, S.; Yu, H.; Shulenin, S.; Hidaka, H.; Kojima, H.; Allikmets, R.; Sakuma, N.; Pegoraro, R.; et al. Identification of a gene, abcg5, important in the regulation of dietary cholesterol absorption. Nat. Genet. 2001, $27,79-83$. [CrossRef] [PubMed]

26. Assmann, G.; Cullen, P.; Erbey, J.; Ramey, D.R.; Kannenberg, F.; Schulte, H. Plasma sitosterol elevations are associated with an increased incidence of coronary events in men: Results of a nested case-control analysis of the prospective cardiovascular münster (procam) study. Nutr. Metab. Cardiovasc. Dis. 2006, 16, 13-21. [CrossRef]

27. Matthan, N.R.; Pencina, M.; LaRocque, J.M.; Jacques, P.F.; D’Agostino, R.B.; Schaefer, E.J.; Lichtenstein, A.H. Alterations in cholesterol absorption/synthesis markers characterize framingham offspring study participants with chd. J. Lipid Res. 2009, 50, 1927-1935. [CrossRef]

28. Rajaratnam, R.A.; Gylling, H.; Miettinen, T.A. Independent association of serum squalene and noncholesterol sterols with coronary artery disease in postmenopausal women. J. Am. Coll. Cardiol. 2000, 35, 1185-1191. [CrossRef]

29. Glueck, C.J.; Speirs, J.; Tracy, T.; Streicher, P.; Illig, E.; Vandegrift, J. Relationships of serum plant sterols (phytosterols) and cholesterol in 595 hypercholesterolemic subjects, and familial aggregation of phytosterols, cholesterol, and premature coronary heart disease in hyperphytosterolemic probands and their first-degree relatives. Metabolism 1991, 40, 842-848. [PubMed]

30. Pinedo, S.; Vissers, M.N.; von Bergmann, K.; Elharchaoui, K.; Lütjohann, D.; Luben, R.; Wareham, N.J.; Kastelein, J.J.; Khaw, K.T.; Boekholdt, S.M. Plasma levels of plant sterols and the risk of coronary artery disease: The prospective epic-norfolk population study. J. Lipid Res. 2007, 48, 139-144. [CrossRef]

31. Fassbender, K.; Lütjohann, D.; Dik, M.G.; Bremmer, M.; König, J.; Walter, S.; Liu, Y.; Letièmbre, M.; von Bergmann, K.; Jonker, C. Moderately elevated plant sterol levels are associated with reduced cardiovascular risk-the lasa study. Atherosclerosis 2008, 196, 283-288. [CrossRef] [PubMed]

32. Wilund, K.R.; Yu, L.; Xu, F.; Vega, G.L.; Grundy, S.M.; Cohen, J.C.; Hobbs, H.H. No association between plasma levels of plant sterols and atherosclerosis in mice and men. Arterioscler Thromb. Vasc. Biol. 2004, 24, 2326-2332. [CrossRef]

33. Weingärtner, O.; Lütjohann, D.; Meyer, S.; Fuhrmann, A.; Cremers, B.; Seiler-Mußler, S.; Schött, H.F.; Kerksiek, A.; Friedrichs, S.; Ulbricht, U.; et al. Low serum lathosterol levels associate with fatal cardiovascular disease and excess all-cause mortality: A prospective cohort study. Clin. Res. Cardiol. 2019, 108, 1381-1385. [CrossRef]

34. Drukker, A.; Levy, E.; Bronza, N.; Stankiewicz, H.; Goldstein, R. Impaired intestinal fat absorption in chronic renal failure. Nephron 1982, 30, 154-160. [CrossRef]

35. Björkhem, I.; Miettinen, T.; Reihnér, E.; Ewerth, S.; Angelin, B.; Einarsson, K. Correlation between serum levels of some cholesterol precursors and activity of hmg-coa reductase in human liver. J. Lipid Res. 1987, 28, 1137-1143. [CrossRef]

36. Kempen, H.J.; Glatz, J.F.; Gevers Leuven, J.A.; van der Voort, H.A.; Katan, M.B. Serum lathosterol concentration is an indicator of whole-body cholesterol synthesis in humans. J. Lipid Res. 1988, 29, 1149-1155. [CrossRef]

37. Baigent, C.; Landray, M.J.; Reith, C.; Emberson, J.; Wheeler, D.C.; Tomson, C.; Wanner, C.; Krane, V.; Cass, A.; Craig, J.; et al. The effects of lowering ldl cholesterol with simvastatin plus ezetimibe in patients with chronic kidney disease (study of heart and renal protection): A randomised placebo-controlled trial. Lancet 2011, 377, 2181-2192. [CrossRef]

38. Lupattelli, G.; Pirro, M.; Mannarino, M.R.; Siepi, D.; Roscini, A.R.; Schillaci, G.; Mannarino, E. Visceral fat positively correlates with cholesterol synthesis in dyslipidaemic patients. Eur. J. Clin. Investig. 2012, 42, 164-170. [CrossRef] [PubMed]

39. Mashnafi, S.; Plat, J.; Mensink, R.P.; Baumgartner, S. Non-Cholesterol Sterol Concentrations as Biomarkers for Cholesterol Absorption and Synthesis in Different Metabolic Disorders: A Systematic Review. Nutrients 2019, 11, 124. [CrossRef]

40. Simonen, P.P.; Gylling, H.; Miettinen, T.A. Body weight modulates cholesterol metabolism in non-insulin dependent type 2 diabetics. Obes. Res. 2002, 10, 328-335. [CrossRef] [PubMed]

41. Simonen, P.; Gylling, H.; Howard, A.N.; Miettinen, T.A. Introducing a new component of the metabolic syndrome: Low cholesterol absorption. Am. J. Clin. Nutr. 2000, 72, 82-88. [CrossRef]

42. Anderson, G.H.; Woodend, D. Effect of glycemic carbohydrates on short-term satiety and food intake. Nutr. Rev. 2003, 61, S17-S26. [CrossRef] 
43. Katan, M.B.; Grundy, S.M.; Jones, P.; Law, M.; Miettinen, T.; Paoletti, R. Efficacy and safety of plant stanols and sterols in the management of blood cholesterol levels. Mayo Clin. Proc. 2003, 78, 965-978. [CrossRef]

44. Kendrick, J.; Chonchol, M.B. Nontraditional risk factors for cardiovascular disease in patients with chronic kidney disease. Nat. Clin. Pract. Nephrol. 2008, 4, 672-681. [CrossRef] [PubMed]

45. Yao, Q.; Pecoits-Filho, R.; Lindholm, B.; Stenvinkel, P. Traditional and non-traditional risk factors as contributors to atherosclerotic cardiovascular disease in end-stage renal disease. Scand. J. Urol. Nephrol. 2004, 38, 405-416. [CrossRef] [PubMed] 